\title{
Epidemiological Profile of Malaria in District Amritsar in Years 2015 and 2016
}

\author{
Shyam Lal Mahajan* \\ Department of Community Medicine, Sri Guru Ram Das Institute of Medical Sciences and Research, India
}

"Corresponding author: Shyam Lal Mahajan, Department of Community Medicine, Sri Guru Ram Das Institute of Medical Sciences and Research, Punjab, India. Tel: +911832870200; +919915589173; 919356005909; Email: slmahajan123@gmail.com

Citation: Mahajan SL (2017) Epidemiological Profile of Malaria in District Amritsar in Years 2015 and 2016. J Community Med Public Health: CMPH-102. DOI: 10.29011/CMPH-102/100002

Received Date: 27 June, 2017; Accepted Date: 21 October, 2017; Published Date: 27 October, 2017

\begin{abstract}
Introduction: Malaria is a mosquito-borne illness. In 2015, 91 countries and areas had ongoing malaria transmission. Between 2010 and 2015, malaria incidence among populations at risk (the rate of new cases) fell by $21 \%$ globally. In that same period, malaria mortality rates among populations at risk fell by $29 \%$ globally among all age groups and by $35 \%$ among children under 5. In 2014, 97 countries and territories had ongoing malaria transmission. The Anopheles mosquito transmits malaria parasites to humans, thrives in warm, tropical and subtropical climates. Malaria situation in India during the years 1996-2013 has shown a declining trend and in years 2011 to 2014 number of malaria cases has decreased in Punjab. A national strategy for malaria elimination named Framework for Malaria Elimination in India 2016-2030 has been developed.
\end{abstract}

Material and Methods: The confirmed cases of malaria were detected by microscopic examination of blood smears in the designated laboratories under National Vector Borne Disease Control Programmed (NVBDCP) in district Amritsar and the epidemiological study of malaria cases recorded in the years 2015 and 2016 was done.

Findings: There were 84 confirmed cases of malaria reported in the years 2015 and 2016 in district Amritsar. Out of these 84 $(58.5 \%)$ cases, 34 (41.5\%) were reported in the year 2015 and 48 in the year 2016. The maximum number of cases $28(34.1 \%)$ of malaria was reported in age group of 21-30 years and no case in age below 2 years. Higher number of male cases was reported than the female cases. Higher total number of rural cases was reported than the urban cases in the years 2016 and 2017. In the years of 2016 higher number of urban cases had been reported while in year 2017 contrary to this, higher number of rural cases was reported. Majority of cases, 62 (77.5\%) had been reported during the period of monsoon (June to September). One case each in month of January, February, November and December (winter) had been reported in the year 2016.

Conclusion: Reporting of maximum number of confirmed cases of malaria in age group 21-30 years, no case in age below 2 years and majority of male cases is consistent with the previous studies. Majority of cases reported during the period of monsoon is also consistent with the previous studies. Reporting of confirmed cases of malaria in the months of January to April, November and December in the district, when a national strategy for malaria elimination has been envisaged is an alarming signal. The entire preventive and control measures for malaria elimination should be taken keeping in view the epidemiological situation.

Keywords: Anopheles Mosquito; Malaria; Malaria Elimination; NVBDCP; Transmission Season

\section{Introduction}

In 2015, 91 countries and areas had ongoing malaria transmission. Between 2010 and 2015, malaria incidence among populations at risk (the rate of new cases) fell by $21 \%$ globally. In that same period, malaria mortality rates among populations at risk fell by $29 \%$ globally among all age groups, and by $35 \%$ among children under 5 [1]. Malaria is an entirely preventable and treatable mosquito-borne illness. In 2014, 97 countries and territories had ongoing malaria transmission. An estimated 3.3 billion people are at risk of malaria, of which 1.2 billion are at high risk. In high-risk areas, more than one malaria case occurs per 1000 population. The malaria-specific Millennium Development Goal has already been met [2]. There are 10 countries with ongoing malaria transmis- 
sion in South-East Asia which accounts for 32 million (15\% of estimated cases worldwide). India, Indonesia, and Myanmar comprise most of the region's reported cases $(94 \%)[3,4]$. Malaria is a leading cause of death for children due to lack of developed immune systems to protect against the disease [3,5]. The Anopheles mosquito, which transmits malaria parasites to humans, thrives in warm, tropical, and subtropical climates [6]. Tens of millions of pregnant women living in endemic regions become pregnant each year [7]. Scale-up of malaria control programs have helped to greatly reduce malaria cases and deaths. While access to both prevention and treatment services has grown over time, gaps remain. $[3,8,9]$

Malaria situation in India during the years 1996-2013 has shown a declining trend. Its Annual Parasite Incidence (API) in year 2005 was 1.68 and has come down to 0.72 in year 2013 [10]. There were 1310656 cases of malaria in India, including 665004 Pf cases and 754 deaths in the year 2011. This was followed in year 2012 by 1067824 cases of malaria including 533695 Pf cases and 519 deaths, in year 2013 by 881730 malaria cases including $463846 \mathrm{Pf}$ cases and 440 deaths and in year 2014 by $1102205 \mathrm{ma}-$ laria cases including Pf 722546 cases and 562 deaths. The number of cases in year 2015 till October 2015 reached 920188 including $6036245 \mathrm{Pf}$ and 245 deaths [11].

There were 2693 cases of malaria in Punjab, including 64 Pf cases and 3 deaths in year 2011. This was followed in year 2012 by 1689 malaria cases including $43 \mathrm{Pf}$ cases and no death, in year 2013 by 1760 malaria cases including 31 Pf cases and no death; and in year 2014 by 1036 malaria cases including 14 Pf cases and no death, in year 2015 till October the number of cases reached to 608 cases including $11 \mathrm{Pf}$ cases and 0 death [11].

A study conducted in district Amritsar showed that there were 140,17, 23, 49 confirmed cases of malaria reported in district Amritsar in years 2011 to 2014. During the years 2011-2014 API of malaria remained below 2 . Study results show that the majority of positive cases were male, in age group of $>14$ years which is consistent with previous studies. Majority of cases $62(77.5 \%)$ had been observed during the period of monsoon (June to September). Similar results had been observed in past studies. All prevention and control measures for malaria should be taken keeping the epidemiological situation in view [12].

Malaria continues to be a major public health problem in South India with majority of cases observed during the period of monsoon and immediately following the end of monsoon (June to October). In 2011, majority of cases were reported in September (28\%) while in 2010 , majority of cases $(23.5 \%)$ were reported in June. A study was conducted from January - September in years 2010 and 2011. A total of 3614 slides were collected from January- September 2010, out of which 136 were positive $(22.8 \%$ P. f.; 77.2\% P.v.). In 2011, a total of 6917 slides were collected, out of which 186 were positive (16.1\% P. f.; $83.9 \%$ P.v.). Posi- tive cases were more frequent in rainy seasons (June-Sept) with a steady increase in the number of cases starting in April [11]. In both 2010 and 2011, majority of positive cases were male (83.8\% and $81.7 \%$, respectively). Majority of positive cases were in the age group of $21-30$ years $(40.44 \%$ and $36.56 \%$, respectively), followed by $11-20$ years $(34.56 \%$ and $27.42 \%$, respectively) in both years [13]. A national strategy for malaria elimination has been envisaged in prompting the development of the National Framework for Malaria Elimination in India 2016-2030. The main focus of this Framework is to propel India on the path towards malaria elimination in a phased manner. Under this Framework, all states/ UTs have been grouped into one of four categories based on their malaria burden. Specific objectives have been established for each of these categories and a mix of interventions will be implemented in each of them.

Eliminate malaria nationally and contribute to improved health, quality of life and alleviation of poverty. In line with the WHO Global Technical Strategy for Malaria 2016-2030 (GTS) and the Asia Pacific Leaders Malaria Alliance Malaria Elimination Roadmap, the goals of the National Framework for Malaria Elimination in India 2016-2030 are: Eliminate malaria (zero indigenous cases) throughout the entire country by 2030; and Maintain malaria-free status in areas where malaria transmission has been interrupted and prevent re-introduction of malaria. The Framework has four

\section{Objectives}

- Eliminate malaria from all 26 low (Category 1) and moderate (Category 2) transmission states/union territories (UTs) by 2022.

- Reduce the incidence of malaria to less than 1 case per 1000 population per year in all states and UTs and their districts by 2024.

- Interrupt indigenous transmission of malaria throughout the entire country, including all high transmission states and Union Territories (UTs) (Category 3) by 2027.

- Prevent the re-establishment of local transmission of malaria in areas where it has been eliminated and maintain national malaria-free status by 2030 and beyond. This Framework will be implemented by the Directorate of National Vector Borne Disease Control Programmer (NVBDCP) which is the umbrella programmed for prevention and control of malaria and five other vector borne diseases [14].

\section{Material and Methods}

The confirmed cases of malaria are detected by microscopic examination of blood smears in the designated laboratories under National Vector Borne Disease Control Programmed (NVBDCP). The laboratories under this programmed in district Amritsar are 
Citation: Mahajan SL (2017) Epidemiological Profile of Malaria in District Amritsar in Years 2015 and 2016. J Community Med Public Health: CMPH-102.

working at the District and Tensile level hospitals, Community Health Centers (CHC), Primary Health Centers (PHC) and the malaria clinics. NVBDCP has included the Medical Colleges of Punjab in the year 2013 for the surveillance of diseases under this programmed. There are two Medical Colleges in district Amritsar namely Government Medical College and Sri Guru Ram Das Institute of Medical Sciences and Research. The confirmation of cases of malaria for both the medical colleges is done at Government Medical College, Amritsar by examination of blood smears. The epidemiological study of confirmed cases of malaria recorded in district Amritsar in the years 2015 and 2016 was done.

\section{Findings}

There were 84 confirmed cases of malaria reported in the years 2015 and 2016 in district Amritsar. Higher number of cases, $48(58.5 \%)$ was reported in year 2016 than 34 (41.5\%) cases in year 2015. (Table 1) shows the age wise distribution of confirmed cases of malaria. The maximum number of cases 28 (34.1\%) of malaria was reported in age group of 21-30 years. No case was reported in the age below 2 years. This was followed by $21(25.6 \%)$, $11(13.4 \%)$ and $11(13.4 \%)$ cases in age groups of 11-20, 31-40 and 41-50 years respectively. Enormous increase in the number of malaria cases, 19 was observed in the age group 21 to 30 years in the year 2016 as compared to 9 cases in the year 2015. The difference in number of cases of malaria reported found in the different age groups had been found insignificant statistically.

\begin{tabular}{|c|c|c|c|c|c|c|}
\hline \multirow{3}{*}{ Age in years } & \multicolumn{4}{|c|}{ Year } & \multirow{2}{*}{\multicolumn{2}{|c|}{ Total }} \\
\hline & \multicolumn{2}{|c|}{2015} & \multicolumn{2}{|c|}{2016} & & \\
\hline & No. & $\%$ & No. & $\%$ & No. & $\%$ \\
\hline $2-5$ & 2 & 5.9 & 1 & 2.1 & 3 & 3.7 \\
\hline $6-10$ & 1 & 2.9 & 2 & 4.2 & 3 & 3.7 \\
\hline 11-20 & 10 & 29.4 & 11 & 22.9 & 21 & 25.6 \\
\hline 21-30 & 9 & 26.5 & 19 & 39.6 & 28 & 34.1 \\
\hline $31-40$ & 6 & 17.6 & 5 & 10.4 & 11 & 13.4 \\
\hline $41-50$ & 4 & 11.8 & 7 & 14.6 & 11 & 13.4 \\
\hline$>\mathbf{5 0}$ & 2 & 5.9 & 3 & 6.2 & 5 & 6.1 \\
\hline Total & 34 & 100.0 & 48 & 100.0 & 82 & 100.0 \\
\hline$X^{2}=3.09$ & \multicolumn{4}{|c|}{ d. f. $=6$} & \multicolumn{2}{|c|}{$p>.05$} \\
\hline
\end{tabular}

Table 1: Age wise distribution.

Table 2 shows the sex wise distribution of confirmed cases of malaria. Higher total number of males' cases, 51 (62.2\%) was reported than the females cases, $31(37.8 \%)$, in the years 2015 and 2016. Both in the individual years of 2015 and 2016 higher number of male cases has been reported than the number of female cases. The difference in number of cases of malaria reported in both the sexes had been found insignificant statistically.

\begin{tabular}{|c|c|c|c|c|c|c|}
\hline \multirow{2}{*}{ Sex } & \multicolumn{4}{|c|}{ Year } & \multicolumn{2}{c|}{ Total } \\
\cline { 2 - 7 } & \multicolumn{2}{|c|}{$\mathbf{2 0 1 5}$} & \multicolumn{2}{c|}{$\mathbf{2 0 1 6}$} & \multicolumn{2}{c|}{} \\
\cline { 2 - 7 } & No. & $\mathbf{\%}$ & No. & \% & No. & $\%$ \\
\hline \multirow{2}{*}{ Male } & 20 & 58.8 & 31 & 64.5 & 51 & 62.2 \\
\hline
\end{tabular}

\begin{tabular}{|c|c|c|c|c|c|c|}
\hline Female & 14 & 41.2 & 17 & 35.5 & 31 & 37.8 \\
\hline Total & 34 & 100.0 & 48 & 100.0 & 82 & 100.0 \\
\hline $\mathbf{X}^{2}=\mathbf{0 . 2 8}$ & d. f. $=\mathbf{1}$ & \multicolumn{4}{|c|}{$\mathbf{p}>\mathbf{0 . 0 5}$} \\
\hline
\end{tabular}

Table 2: Sex wise distribution.

Table 3 shows the area wise distribution of confirmed cases of malaria. Higher total number of rural cases, 50 (60.1\%) was reported than the urban cases, 31 (37.8\%), in the years 2016 and 2017. In the years of 2016 higher number of urban cases 16 (47.1\%) has been reported while in year 2017 contrary to this, higher number of rural cases34 (70.8\%) had been reported. The difference in number of cases of malaria reported found in both the rural and urban areas had been found significant statistically.

\begin{tabular}{|c|c|c|c|c|c|c|}
\hline \multirow{3}{*}{ Area } & \multicolumn{4}{|c|}{ Year } & \multirow{2}{*}{\multicolumn{2}{|c|}{ Total }} \\
\hline & \multicolumn{2}{|c|}{2015} & \multicolumn{2}{|c|}{2016} & & \\
\hline & No. & $\%$ & No. & $\%$ & No. & $\%$ \\
\hline Urban & 18 & 52.9 & 14 & 29.2 & 32 & 39.0 \\
\hline Rural & 16 & 47.1 & 34 & 70.8 & 50 & 60.1 \\
\hline Total & 34 & 100.0 & 48 & 100.0 & 82 & 100.0 \\
\hline $\mathrm{X}^{2}=4.75$ & \multicolumn{4}{|c|}{ d. f. $=1$} & \multicolumn{2}{|c|}{$P<0.05$} \\
\hline
\end{tabular}

Table 3: Area wise distribution.

Table 4 shows the month wise distribution of confirmed cases of malaria. Majority of cases, $62(77.5 \%)$ had been reported during the period of monsoon (June to September). Maximum number of cases, $28(34.15 \%)$ in the years 2015 and 2016 had been found in September. This was followed by $20(24.39 \%)$ in the August and the minimum number of cases, $1(1.22 \%)$ in each month of November and December had been found. No confirmed case of malaria had been reported in the months of January to April in the year 2015 while $9(11.0 \%)$ cases had been reported in the same months of winter season in the year 2016. This transmission of malaria in winter season is an alarming signal. The difference in number of cases of malaria reported found in different months of the year had been found insignificant statistically.

\begin{tabular}{|c|c|c|c|c|c|c|}
\hline \multirow{2}{*}{ Month } & \multicolumn{4}{|c|}{ Year } & \multicolumn{2}{|c|}{ Total } \\
\cline { 2 - 6 } & \multicolumn{2}{|c|}{$\mathbf{2 0 1 5}$} & \multicolumn{2}{|c|}{$\mathbf{2 0 1 6}$} & \multicolumn{2}{|c|}{} \\
\cline { 2 - 6 } & No. & $\mathbf{\%}$ & No. & $\mathbf{\%}$ & No. & \% \\
\hline January & 0 & 0.00 & 1 & 2.08 & 1 & 1.22 \\
\hline February & 0 & 0.00 & 1 & 2.08 & 1 & 1.22 \\
\hline March & 0 & 0.00 & 2 & 4.17 & 2 & 2.44 \\
\hline April & 0 & 0.00 & 5 & 10.42 & 5 & 6.10 \\
\hline May & 1 & 2.94 & 1 & 2.08 & 2 & 2.43 \\
\hline June & 5 & 14.71 & 3 & 6.25 & 8 & 9.76 \\
\hline
\end{tabular}


Citation: Mahajan SL (2017) Epidemiological Profile of Malaria in District Amritsar in Years 2015 and 2016. J Community Med Public Health: CMPH-102.

\begin{tabular}{|c|c|c|c|c|c|c|}
\hline July & 3 & 8.82 & 3 & 6.25 & 6 & 7.32 \\
\hline August & 11 & 32.35 & 9 & 18.75 & 20 & 24.39 \\
\hline September & 10 & 29.41 & 18 & 37.50 & 28 & 34.15 \\
\hline October & 3 & 8.82 & 4 & 8.33 & 7 & 8.54 \\
\hline November & 0 & 0.00 & 1 & 2.08 & 1 & 1.22 \\
\hline December & 1 & 2.94 & 0 & 0.00 & 1 & 1.22 \\
\hline Total & 34 & 100.00 & 48 & 100.00 & 82 & 100.00 \\
\hline$X^{2}=12.091^{a}$ & \multicolumn{4}{|c|}{ d. f. $=11$} & \multicolumn{2}{|c|}{$P>0.05$} \\
\hline
\end{tabular}

Table 4: Month wise distribution

There are 7 rural blocks in district Amritsar as shown in the Table 5 this table shows the rural block wise distribution of confirmed cases of malaria. Out of the total 82 confirmed cases of malaria reported in district Amritsar 49 (59.76\%) cases had been reported from its rural blocks. Majority of the cases $32(65.3 \%)$ had been reported from Lopoke, Manvel and Ramada's blocks.

\begin{tabular}{|c|c|c|c|c|c|c|}
\hline \multirow{2}{*}{ Block } & \multicolumn{4}{|c|}{ Year } & \multicolumn{2}{c|}{ Total } \\
\cline { 2 - 7 } & \multicolumn{2}{|c|}{$\mathbf{2 0 1 5}$} & \multicolumn{2}{c|}{$\mathbf{2 0 1 6}$} & \multicolumn{2}{c|}{} \\
\cline { 2 - 7 } & No. & \% & No. & \% & No. & \% \\
\hline Bababakala & 2 & 12.5 & 1 & 3.0 & 3 & 6.1 \\
\hline Lopoke & 4 & 25.0 & 6 & 18.2 & 10 & 20.4 \\
\hline Manawala & 6 & 37.5 & 5 & 15.2 & 11 & 22.4 \\
\hline Ramdas & 3 & 18.75 & 8 & 24.2 & 11 & 22.4 \\
\hline Tarsika & 0 & 0.00 & 4 & 12.1 & 4 & 8.2 \\
\hline Threawal & 0 & 0.00 & 4 & 12.1 & 4 & 8.2 \\
\hline Verka & 1 & 6.25 & 5 & 15.2 & 6 & 12.2 \\
\hline Total & 16 & 100.0 & 33 & 100.0 & 49 & 100.0 \\
\hline $\mathbf{X}^{2}=\mathbf{8 . 9 4}$ & d. f. $=\mathbf{6}$ & & \multicolumn{3}{c|}{ P $<\mathbf{0 . 0 5}$} \\
\hline
\end{tabular}

Table 5: Rural block wise distribution.

\section{Conclusion}

The maximum number of cases 28 (34.1\%) of malaria was reported in age group of 21-30 years and majority of cases were males. No case was reported in the age below 2 years. The study is consistent with the previous studies [12,15-17]. Majority of cases, $62(77.5 \%)$ had been reported during the period of monsoon (June to September). Similar results had been found in the previous studies $[12,13]$. Reporting of confirmed cases of malaria in the months of January to April and December (in winter season) in the district, when a national strategy for malaria elimination has been envisaged is an alarming signal. Similar results had been observed in past studies $[12,13]$. The entire preventive and control measures for malaria elimination should be taken keeping in view the epidemiological situation.

\section{Limitation}

There is under-reporting of cases as the cases reported were mainly from the designated laboratories and there was non-involvement of many of the private health care institution laboratories and many health clinics having no laboratories.

\section{Acknowledgements}

The author acknowledges the thanks of Mr. Kartikay Mahajan who helped in making the tables and applying the statistical tests, Dr. Madan Mohan, District Epidemiologist and Manjit Singh Health Assistant in Civil Surgeon's Office, Amritsar, who made discussions with the author.

\section{Additional information}

(i) Source of funding: Self

(ii) Ethical clearance: Not needed as the study is based on the NVBDCP/IDSP programs.

(iii) Reference in Vancouver style

\section{References}

1. World Health Organization (2017) Malaria fact sheet updated.

2. World Health Organization (2014) Fact sheet on the world malaria report.

3. US. Global health policy 2014 The global malaria epidemic Fact sheet 2013 Mar.

4. WHO. World malaria report 2012.

5. Tanner M, de Savigny D (2008) Malaria eradication back on the table.

6. CDC. Malaria.

7. Dellicour S, Tatem AJ, Guerra CA, Snow RW, terKuile FO (2010) Quantifying the number of pregnancies at risk of malaria in 2007. PLoS Medicine 7: 1.

8. United Nations. The millennium development goals report 2011

9. Roll Back Malaria. The Global Malaria Action Plan 2008.

10. Government of India (2014-15) Ministry of Health and Family Welfare, National Vector Borne Disease Control Programme. Annual report.

11. Government of India, Ministry of Health and Family Welfare, National Vector Borne Disease Control Programme. Malaria situation in India 2015 and 2016.

12. Mahajan SL (2016) Epidemiological profile of malaria: Analysis in district Amritsar. Indian Journal of Public Health Research and Development.

13. Kamath R, Gaitonde S, Tripathi P, Das D, Banerjee M, Shetty M et al. (2012) Clinico-epidemiological profile of malaria: Analysis from a primary health centre in Karnataka, Southern India. Global journal of medicine and public health1: 1-6.

14. Government of India, Ministry of Health and Family Welfare, National Vector Borne Disease Control Programme (2016-2030) National framework for malaria elimination in India xii, 1

15. Gurang B, Bairy I, Jagadishchandra, Manohar C (2010) Evaluation of falcivax against quantitative buffy coat (QBC) for the diagnosis of malaria. International Journal of collaborative Research on Internal Medicine \& Public Health.

16. Chowta MN, Chowta KN (2007) Study of clinical profile of malaria at KMC Hospital, Attavar, India. Journal of Clinical and Diagnostic Research 1: 110-115

17. Talawar AS, Pujar S (2011) Epidemiological situation of malaria in irrigated areas: A case study of Raichur district, Karnataka. Karnataka Journal of Agricultural Sciences 24: 198-200. 